\title{
Pharmacist Led Assessment of Medication Inappropriateness in a Medicine Department of a Tertiary Care Hospital
}

\author{
Inamdar Syed Ziaa,*, Sunanda Nandikol', Pradeepthi Katragadda', Raghavendra Vinayakarao \\ Kulkarni ${ }^{1}$, Kusal Das ${ }^{2}$, Rucha Kulkarni' ${ }^{1}$, Rashmi ${ }^{3}$, Zulfeqar ${ }^{3}$, Gautam ${ }^{3}$ \\ 'Department of Pharmacy Practice, BLDEA's SSM College of Pharmacy and Research Centre, Vijayapura, Karnataka, INDIA. \\ ${ }^{2}$ Department of Physiology, Shri BM Patil Medical College Hospital and Research Centre, Vijayapura, Karnataka, INDIA. \\ ${ }^{3}$ Pharm D Intern, BLDEA's SSM College of Pharmacy and Research Centre, Vijayapura, Karnataka, INDIA.
}

\begin{abstract}
Background and Introduction: Inappropriate use of drugs is a major problem which contributes towards morbidity, mortality and increased length of hospital stay among patient population. Prescription inappropriateness is significant contributor for adverse health and economic outcome and retard quality use of medication among patient population. Objectives: To assess appropriateness of medication prescribed among study population by applying Medication Appropriateness Index (MAI). Methods: A prospective observational study was carried out in medicine department [emergency and general] of a teaching hospital. Patient pertinent and prescription medicine information were reviewed and assessed using medication appropriateness index to intercept medication inappropriateness. Results: Around 1863 drugs were prescribed of which appropriate drugs are found to be 1639 $(87.97 \%)$ and inappropriate drugs were found to be $424(22.76 \%)$ based on MAl scoring. Out of 424 Inappropriate drugs, $276(65.17 \%)$ were marginally inappropriate, $44(10.26 \%)$ were inappropriate and $104(24.55 \%)$ drugs were with maximal inappropriateness. Polypharmacy was identified as the major factor contributing towards medication inappropriateness 107 (43.145\%). Conclusion: The study shows that drug therapy is not consistent on prescribing principles of medicine appropriateness scale and notices the importance and scope of prescriber and patient education. Clinical pharmacist led review of drug therapy assessment can enhance prescribing quality.
\end{abstract}

Key words: Medication appropriateness index, Inappropriate prescribing, Likert 3-point scale, Drug-drug interaction, Clinical pharmacist, Polypharmacy.

\section{INTRODUCTION}

Prescription Inappropriateness is termed as failing to attain the maximum quality of drug use. It is also outlined as the prescription that introduced an important menace of adverse drug associated incident while there is a scope for an equally or more effective substitute medication. ${ }^{1}$ Literature had showed that the inappropriate medicines practice is very common in general teaching hospital in most part of the developing and developed world. ${ }^{1}$ Universally it is observed that $50 \%$ of the patients fails to take their medications correctly due to inappropriate prescribing, dispensing and usage, while other half of the patients fail to follow up the therapy. ${ }^{2}$
The Medication Appropriateness Index (MAI) has many benefits for assessing Inappropriate Prescribing (IP) when compared to that of medications preference among patient population. It is well-defined and hypothetically delicate to distinguish significant IP, or disease; it explains multiple factors of prescribing appropriateness, so this can be applied on the basis of patient's medical history. MAI can be tested in both inpatient and ambulatory care. An additional improvement in its rationality and consistency and in-detailed information was also updated in the recent version. ${ }^{3,4}$ The MAI is intended to agree to rating of ten explicit criteria to establish whether a given medication is appropriate for an individual.
DOI: 10.5530/ijopp.12.4.55

Address for correspondence: Dr. Inamdar Syed Zia, Professor and Clinical Pharmacist,

Department of Pharmacy Practice, BLDEA's SSM College of Pharmacy and Research Centre Vijayapura-586103, Karnataka, INDIA.

Phone no: +91-9986666016 Email Id: syedzia.inamdar@ gmail.com

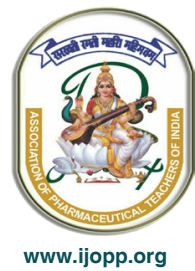


The ten criteria of the MAI, worded as questions, relate to the individual patient and drug in question. These criteria are: An indication for the drug; drug effectiveness for the patient's condition; correct dosage and directions; practical directions; drug-drug interaction; drug-disease interaction; unnecessary duplication with other drugs; duration of therapy and cost-effectiveness. Each criterion for the appropriateness of prescribing is rated on a threepoint Likert scale, depending on whether the drug is appropriate, marginally appropriate or not appropriate.

The pharmacist's role has advanced considerably from bench side to bed side with the delivery of pharmaceutical care to enhance appropriate medication use and minimizing actual and potential medication related problems. Medication appropriateness coalesce varied fundamentals of evidence-based practice with professional judgment when evidence is incomplete. Pharmaceutical care activities performed by clinical pharmacists as a part of multidisciplinary health care team promotes medication use optimization and improve treatment outcomes in different clinical settings, including hospital wards, intensive care units and in community. However, the literature is inadequate in reporting studies regarding models of practice that involve clinical pharmacists.

The Emergency Department (ED) are usually a busy environment in teaching hospitals and pose challenge for optimum delivery of care. The risk of suboptimal medication use varies in inpatients to ambulatory setting but higher in ED patients. Detection and interception of these inappropriate prescribing highlight the need for pharmacist led deliverance of pharmaceutical care services in these setting and could be the next step practice model for advance pharmacy practice in health care institution. Therefore, the present study is an attempt to apply the MAI by a clinical pharmacist to identify issues of inappropriate prescribing amongst patients admitted to ED and medicine ward. ${ }^{5}$

\section{MATERIALS AND METHODS}

A prospective observational study was conducted over a six-month period in a tertiary care hospital in Bijapur city of north Karnataka. All patients aged 18 years and over, taking one or more medicines, who presented at the emergency and general medicine ward during the study period under the care of participating consultant physicians team were included in the study. The sixmonth time frame allowed sensible time duration to study a meaningful sample size. The study was approved by the Ethics Committee and confidentiality of the subjects was maintained. This study involved a cluster sampling method of patients admitted under participating consultant's team.

The sample size was estimated from previous studies which used the MAI. Taking a level of confidence of $95 \%$ and margin of error of 10 , the sample population was estimated to be that of 210 patients. Following the medical assessment after admission at selected wards the patient pertinent clinical and demographic information, medication treatment chart, patient case notes from files were reviewed by the pharmacist. Treatment review of both acute and chronic medication a patients was on were performed afterward rounds to detect medication inappropriateness and the necessary data was documented on a standard data collection form. Every medication in the treatment chart of the study subject were evaluated and verified for its appropriateness using MAI.

\section{RESULTS}

A total of 210 [male $56.6 \%$ and female $43.3 \%$ ] subjects were finally reviewed in the study of which 110 (52.3\%) patients were enrolled from ED and 100 (47.6\%) from medicine ward. The minimum and maximum age of the enrolled subjects ranged from 18 to 80 years and above. Majority of the subjects were elderly $64(58.18 \%)$ and were from ED followed by other younger age groups as presented in Table 1.

A total of 1863 drugs were prescribed in all 210[8.88 drugs per patient]. The majority of the drug prescribed class was of cardiovascular category $(26.15 \%)$ followed by other major classes as illustrated [Figure 1].

Around $424(22.76 \%)$ prescribed drug were found to be inappropriate based on MAI scoring. Out of 424

Table 1: Summary characteristics of study population.

\begin{tabular}{cc} 
Characteristics & $\boldsymbol{N}$ \\
\hline Total & 210 \\
Enrolled from Emergency ward & 110 \\
Enrolled from Medicine ward & 100 \\
Gender & Male (119) \\
& Female (91) \\
Age range & $18-85$ years \\
Age Group (nos) & $18-39(25)$ \\
& $40-59(74)$ \\
& $60-79(94)$ \\
Diagnosis and Co-morbidities & $\geq 80(17)$ \\
Total drugs prescribed & 544 \\
Range of drug & 1863 \\
Average drug per patient & $1-20$ \\
\hline
\end{tabular}


Inappropriate drugs, $276(65.17 \%)$ were marginally inappropriate, $44(10.26 \%)$ were inappropriate and $104(24.55 \%)$ drugs were found to be with maximal inappropriateness [Table 2]. Drug-drug interaction (50\%), drug therapy duration (16.99\%), duplication $(7.31 \%)$ and drug indication $(8.01 \%)$ are some of the major MAI criterion categories identified with inappropriateness [Table 3 and Figure 2]. An additional beer criterion-based drug inappropriateness assessment in elderly patient only revealed 5.80\% drugs as inappropriate [Table 4].

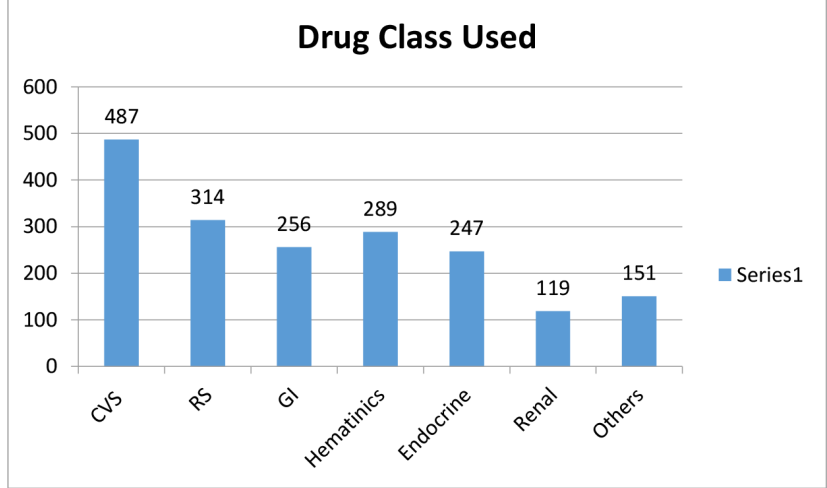

Figure 1: Distribution of Drug classes used among study population.

CVS: Cardiovascular system, RS: Respiratory system, GI: gastrointestinal

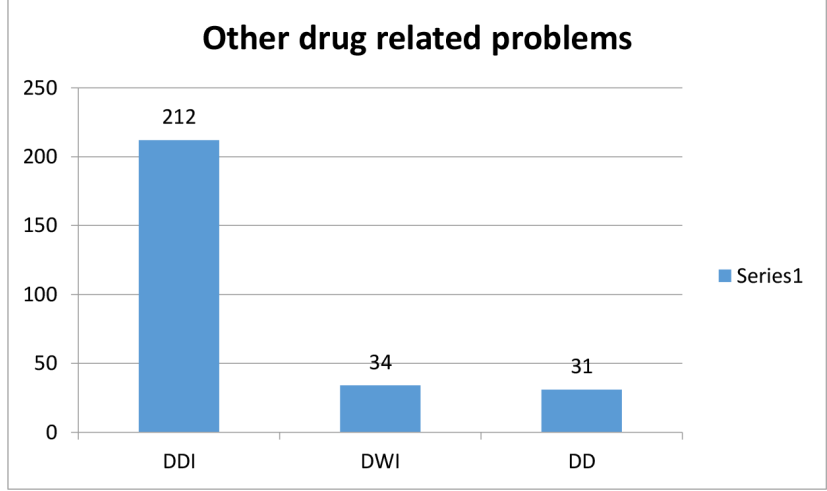

Figure 2: Other drug related problems reported.

DDI: drug-drug interaction, DWI: drug without indication, DD: drug duplication

\section{Table 2: Distribution of Inappropriateness $n=424$.}

\begin{tabular}{ccc} 
Inappropriateness category & Frequency & $\%$ \\
\hline Marginally inappropriate (5-8) & 276 & 65.17 \\
Inappropriate (9-12) & 44 & 10.26 \\
Maximal inappropriate (13-18) & 104 & 24.55 \\
\hline
\end{tabular}

Table 3: Distribution of Inappropriateness as MAI criterion.

MAl criterion Question

Drugs Inappropriateness $n=424$

\begin{tabular}{ccc} 
& \multicolumn{2}{c}{$\boldsymbol{n = 4 2 4}$} \\
\cline { 2 - 3 } & $\boldsymbol{n}$ & \% \\
\hline Drug indication & 34 & 8.01 \\
Effectiveness & 10 & 2.36 \\
Correct dosage & 10 & 2.36 \\
Correct direction & 5 & 1.18 \\
Practical directions & 6 & 1.41 \\
Drug- Drug Interaction & 212 & 50 \\
Drug - Disease Interaction & 12 & 2.83 \\
Duplication & 31 & 7.31 \\
Duration of Therapy & 72 & 16.99 \\
Expense & 32 & 7.55 \\
\hline
\end{tabular}

Table 4: Inappropriate Drug as per Beers criteria (only in elderly) $n=108$.

\begin{tabular}{ccc} 
Drug & Frequency & $\%$ \\
\hline Nitrates & 22 & 20.37 \\
Alprazolam & 19 & 17.59 \\
Insulin & 17 & 15.74 \\
Aspirin & 11 & 10.15 \\
Sulfonylureas & 10 & 9.25 \\
Zolpidem & 06 & 5.55 \\
Chlorpheniramine & 05 & 4.62 \\
Spironolactone & 04 & 3.70 \\
Diclofenac & 03 & 2.77 \\
Pioglitazone & 03 & 2.77 \\
Pentazocine & 02 & 1.85 \\
Digoxin & 02 & 1.85 \\
Nitroglycerin & 02 & 1.85 \\
Cyproheptadine & 01 & 0.92 \\
Amitryptylline & 01 & 0.95 \\
\hline
\end{tabular}

The most common inappropriate drug classes were Benzodiazepines 108 (25.44\%) followed by Antibiotics 68 (16.07\%), Anti hypertensive's 45 (10.71\%), antidiabetics 44 (10.26\%), NSAIDS 40 (9.35\%), Diuretics 36 (16.07\%), antiemetics 32 (7.58\%), opioid analgesics $17(4.01 \%)$ and others $34(8.03 \%)$ [Table 5].

Of the contributing factors possibly reported for medication inappropriateness, Polypharmacy was found to be the major factor 107 (43.145\%), followed by duration of therapy $40(16.12 \%)$, personnel errors were $28(11.29 \%)$ etc [Table 6]. 
Table 5: Most Common Drug class with Inappropriateness, $n=424$.

\begin{tabular}{ccc}
\hline Drugs & Frequency & Percentage \\
\hline Benzodiazepines & 108 & 25.44 \\
Antibiotics & 68 & 16.07 \\
Anti-Hypertensive's & 45 & 10.71 \\
Diabetic drugs & 44 & 10.26 \\
NSAIDS & 40 & 9.35 \\
Diuretics & 36 & 8.42 \\
Anti-emetics & 32 & 7.58 \\
Opioid analgesics & 17 & 4.01 \\
Others & 34 & 8.03 \\
\hline
\end{tabular}

Table 6: Contributing Factors for Medication Inappropriateness, $n=448$.

\begin{tabular}{ccc} 
Factor & Frequency & Percentage \\
\hline Polypharmacy & 193 & 43.15 \\
Duration of therapy & 72 & 16.13 \\
Inappropriate & 51 & 11.29 \\
communication & & \\
Dosage form errors & 47 & 10.48 \\
Inappropriate therapy & 40 & 8.87 \\
Therapeutic duplication & 31 & 6.85 \\
Other factors & 14 & 3.22 \\
\hline
\end{tabular}

\section{DISCUSSION}

MAI is implicit tool criteria for the use in elderly patients initially. However, it can be utilized in all age groups as no statistical difference was observed with reliability of result in different age groups. A total of 210 subjects were finally reviewed and included in the study, among them 119 (56.6\%) were male and 91 (43.3\%) were female. Majority of the patients were elderly with higher admission pattern observed both in ED and medicine ward against non-elderly suggestive of their susceptibility to disease chronicity and ageing process and are more likely to utilized ED level of services. ${ }^{6}$

A total of 1863 drugs were prescribed in 210 study subjects. The inappropriate drugs were found to be 424 $(22.76 \%)$ based on MAI scoring and 1439 (77.24\%) appropriate drugs, Among the appropriate, drugs with score 0 were $45.57 \%$, drugs with score 1 were $11.10 \%$, drugs with score 2 were $18.05 \%$, with score $3(16.71 \%)$ and score 4 were $8.54 \%$ based on MAI scale.

Among 424 Inappropriate drugs $65.1 \%$ were marginally inappropriate, $10.26 \%$ were inappropriate and $24.55 \%$ drugs were found to be with maximal inappropriateness based on the MAI scoring.

Indian Journal of Pharmacy Practice, Vol 12, Issue 4, Oct-Dec, 2019
The study reveals $22.76 \%$ prescribed drugs were inappropriate for one or the other reason. Untoward adverse drug event, deficient or underutilization of health care services, risk of morbidity and mortality are some of the outcomes which are quite common because of inappropriate medication use. ${ }^{7}$ Inappropriate prescription reporting using MAI varied from 39.5\% to $78.3 \%$ at varied level of health care setting possibly because of difference in study design. ${ }^{8-10}$ More than $90 \%$ of the study subjects had one or more medications rated as inappropriate by MAI tool, as quite similar to reported studies. ${ }^{8-10}$ Inadequate prescribing practice has been associated with reduced update on clinical pharmacology and therapeutics. These inadequacies further exaggerated by deficient system design, lack of teamwork and environmental stress impact negatively on prescribing practices. ${ }^{11}$

The major category of inappropriate prescribing was observed with drug- drug interaction which shares $50 \%$ of all inappropriateness reported followed by duration of therapy $16.99 \%$, drug indication $8.01 \%$ and duplication of therapy $7.31 \%$. These occur possibly with multiple use of medication or polypharmacy. An important component probably is communication among health care professional treating the patient and also communication between health care professional and patient/ patient attendees, if the instruction were not conveyed appropriately might lead to cause drug use error. This might bring in the need of a system or mechanism which encourages writing written instructions and appropriate verbal mode. Duration of therapy $16.99 \%$ was the second major inappropriate category observed in the study, there are some plausible clinical situations where the drug therapy duration was not adhered owing to peer pressure based on gathered local evidence and moreover at times when benefit outweighs risk but it was observed that lack of therapeutics update as possible reason for such occurrence. The study reveals around $8.01 \%$ of episodes where there was a problem related with the use of drug without any indication in the patients. The issues of drug without indication were attributed to deficient patient medical/medication history assessment and deficient documentation of the findings. Medication with look alike, sound alike and name alike are possible reason for drug without indication problems. These also contribute towards drug duplication. Branded medicines and their substitution during dispensing precipitate drug duplication problem observed among the study population.

The drug like nitrates (20.37\%), alprazolam (7.59\%), insulin (5.74\%), aspirin (10.15\%) and sulphonylureas $(9.25 \%)$ are chiefly involved as inappropriate prescribing 
reported through Beers criteria assessment among the elderly subject of the study population [Table 4] also, as reported through MAI, Benzodiazepines (25.4\%), Antibiotics (16.07\%), anti-Hypertensive's (10.71\%) and anti-diabetic drugs $(10.26 \%)$ were majorly encountered with inappropriate prescribing. The observed therapeutic classes were different from study to study. ${ }^{8,12,13}$

The observation reported of the study are of significance as they provide the need to rationalize and improve the prescribing efficiency by approaches of following guidelines, implementing protocol and safe drug use policies and continued education to all health care professionals involved in the deliverance of patient care. The approaches might reduce the occurrence of medication related injury and could possibly have a costeffective impact on overall treatment cost. The vigilant role of pharmacist at bed side might help prevent a potential problem from becoming an actual problem and promote safe drug use practice in a clinical setting and might optimize overall treatment outcome

The MAI was applied to detect inappropriate prescriptions; though it's marked utility it does not address issues like adverse drug reactions assessment and medication adherence. However, MAI satisfactorily assisted pharmacists in detection of medication inappropriateness

\section{CONCLUSION}

The study reveals the significant role of a clinical pharmacist to monitor drug therapy. Despite the existence of comprehensive guidelines for use and monitoring of appropriate use of drug, these parameters have not been performed accurately. The lack of acquaintance and update of prescribers with the guidelines may be a potential reason for not administering drug appropriately. Most of the subjects with inappropriate administration observed were mostly in geriatrics patients. Use of drug in geriatric patients must be carefully monitored. The study concluded that drug therapy was moderately consistent with clinical principles and perceives the opportunity and significance of prescriber education.

\section{ACKNOWLEDGEMENT}

The authors are thankful to the management of BLDE association and hospital for supporting the work.

\section{CONFLICT OF INTEREST}

The authors declare no conflict of interest.

\section{ABBREVIATIONS}

MAI: Medication Appropriateness Index; IP: Inappropriate prescribing; ED: Emergency department; NSAIDs: Non-steroidal anti-inflammatory drugs.

\section{SUMMARY}

Increased morbidity, mortality and extension of hospitalization have also been attributed to inappropriate drug use among patient population and is considerably an important risk factor influencing patient outcomes. The studies approaches to assess prescription appropriateness using Medication Appropriateness Index (MAI) scale and observed significant gaps in prescribing quality though unintentional and are attributed to deficient clinical update and acquaintance to standard therapeutic guidelines. The study notices the importance and scope of prescriber and patient education.

\section{REFERENCES}

1. Hogerzeil HV. Promoting rational prescribing: An international perspective. British Journal of Clinical Pharmacy. 1995;39(1):1-6.

2. The rational use of drugs. Report of the Conference of Experts. Geneva. World Health Organization. 1985.

3. JrManasse HR. Medication use in an imperfect world: Drug misadventuring as an issue of public policy, part 1. Am J Hosp Pharm. 1989;46(5):929-44.

4. Linnebur RLSA, Bryant LL, Ruscin JM. Inappropriate prescribing in the hospitalized elderly patient: Defining the problem, evaluation tools and possible solutions. Clin Interv Aging. 2010;5:75-87.

5. West LM, Cordina M, Cunningham S. Clinical pharmacist evaluation of medication inappropriateness in the emergency department of a teaching hospital in Malta. Pharmacy Practice. 2012;10(4):181-7.

6. Strange GR, et al. Use of emergency departments by elderly patients: Projections from a Multicenter data base. Annals of Emergency Medicine. 1992;21(7):819-24.

7. Hamilton JH, Gallagher PF, O'Mahony D. Inappropriate prescribing and adverse drug events in older people. BMC Geriatr. 2009;9(1):5.

8. Hanlon JT, Artz MB, Pieper CF, Linblad Cl, Sloane RJ, Ruby CM, et al. Inappropriate medication use among frail elderly inpatients. Ann Pharmacother. 2004;38(1):9-14.

9. Bregnhoj L, Thirstrup S, Kristensen MB, Bierrum L, Sonne J. Prevalence of inappropriate prescribing in primary care. Pharm World Sci. 2007;29(3):109-15

10. Pallant J. SPSS survival manual: A step by step guide to data analysis using SPSS. $4^{\text {th }}$ edition. England: Open University Press. 2010.

11. Kaushal R, Bates DW, Landrigan C, McKenna KJ, Clapp MD, Federico F, et al. Medication errors and adverse drug events in pediatric inpatients. JAMA. 2001;285(16):2114-20.

12. Perri M, Menon AM, Deshpande AD, Shinde SB, Jiang R, Cooper JW, et al. Adverse Outcomes Associated with Inappropriate Drug Use in Nursing Homes. Ann Pharmacother. 2005;39(3):405-11

13. Schmader K, Hanlon JT, Weinberger M, Landsman PB, Samsa GP, Lewis I, et al. Appropriateness of medication prescribing in ambulatory elderly patients. $J$ Am Geriatr Soc. 1994;42(12):1241-7. 